\title{
Diagram for the diagnosis of SARS-CoV-2: a view with a process perspective
}

\author{
Ana Paula Melo Mariano - paulamariano@uesc.br \\ State University of Santa Cruz \\ https://orcid.org/0000-0002-3177-7790
}

Pedro Costa Campos Filho - pcfilho@uesc.br

State University of Santa Cruz

https://orcid.org/0000-0001-6099-5634

Simone Borges Simão Monteiro - simoneborges@ unb.br

University of Brasilia

https://orcid.org/0000-0001-5546-8143

Ari Melo Mariano - arimariano@unb.br

University of Brasilia

https://orcid.org/0000-0002-7987-5015

\begin{abstract}
Many studies have been developed by the scientific and medical community to learn more about COVID-19. Although studies have advanced, it is common to note that there are still some misconceptions, especially as the information moves from scientific to widespread use in hospitals, clinics, laboratories, and especially the population. Thus, the objective of this study was to present the testing process for the detection of SARS-CoV2 in the patient, paying attention to the key dates and possible diagnoses. To achieve this goal, a process diagram was founded to establish the path of detection and diagnosis. Also, a table for interpreting the results has been prepared. The results collaborate with a clearer understanding of the diagnostic process and expand the understanding to other areas, mainly those that need binary inputs to contribute to the fight against the virus, such as computing, mathematics, and engineering.
\end{abstract}

Keywords: SARS-CoV-2, COVID-19, Process Diagram, Detection.

\section{Authors' contribution:}

APMM conceptualized, designed, collected data, provided information and developed the study. The PCCF conceptualized, designed, collected data, provided information and prepared the study. SBSM developed the process diagram, provided information and prepared the study. The AMM, organized the functions, promoted the discussion, collected data, reviewed the methodological issues and prepared the study. All authors approved the final version of the manuscript. 


\section{Introduction}

According to Wang et al. (2020), the outbreak of COVID-19 was unique in its speed of transmission, becoming a global health emergency in just a few weeks. The number of COVID-19 cases confirmed and suspected, increased rapidly, reaching 9,296,202 cases and 479,133 confirmed deaths on June 25, 2020 (WHO, 2020a). In Brazil, the first case has been reported on February 26, 2020, with the first fatal victim on March 17, 2020, both in São Paulo (Kiernan \& Bardi, 2020). The country currently has 1,145,906 cases and 52,645 confirmed deaths (WHO, 2020a).

The vertiginous growth of the virus and the number of deaths generated a global commitment by the medical and scientific community to understand more about the SARS-CoV-2 virus and the disease caused by it, COVID-19 (Le et al. 2020; Mehta et al. 2020; Rothan et al. 2020; Randhawa et al. 2020)

Although many discoveries have been made to the (Velavan et al. 2020; Zheng et al. 2020; WHO, 2020b), many points still raise doubts, and many preliminary results still require counter-proofs to be considered suitable for adoption.

However, with the advent of the internet and social networks, much information about the virus was disseminated more quickly and extensively when compared to other similar virus outbreaks, such as SARS, in 2003 (Zhu et al. 2020). What could be something beneficial, contributed to an increase in uncertainty, because before this information might be better debated in the scientific field, it had already reached the public, often mistaken or incomplete.

Ho, et al. (2020), explain the initial difficulty in understanding the characteristics of the virus in terms of transmission and virulence, further increasing uncertainty. Although considerable progress has been made regarding the virus transmission and detection process, there are still many doubts regarding the virus detection process to the detriment of the available options. Thus, this work aims to present the testing process for the detection of SARS-CoV-2 in the patient, paying attention to the key dates and possible diagnoses.

\section{Methods}

The research was exploratory with a qualitative approach through bibliographic research and comparison with protocols performed in laboratories and hospitals. Interpretation sessions have carried out on the different sources and applied knowledge of health professionals.

The team executing this work was formed by professionals from different areas, each with their contribution. A Ph.D. in science with training in pharmacy, hematology, and clinical analysis, responsible for interpreting the exams. A Doctor in biology and biotechnology of microorganisms, trained in biomedicine, responsible for studying the behavior of the virus as a biological agent. A Ph.D. in production engineering with training in chemical engineering and process specialist, responsible for the procedural vision and a Post-Doctor in scientific methodology with training in administration, 
responsible for the linear organization of the document and consolidation of the information. Bizagi software was used to produce the process diagram.

For a better view of the figure (https://drive.google.com/file/d/10Y75nqet9eY2MwIf4mDNIn81JGEfTe4/view? usp=sharing?).

If you want to see the study in Portuguese (https://drive.google.com/file/d/1MNQ2VfkltOYMAdtLBDJ5zlZNr7h47xb/view?usp=sharing).

\section{Results and Analysis}

Figure 1 shows the testing process for COVID-19. The individual should be evaluated before being subjected to the collection of SARS-CoV-2 diagnostic tests. The process describes two paths, one that represents the symptomatic individual, and the other for asymptomatic individuals. 


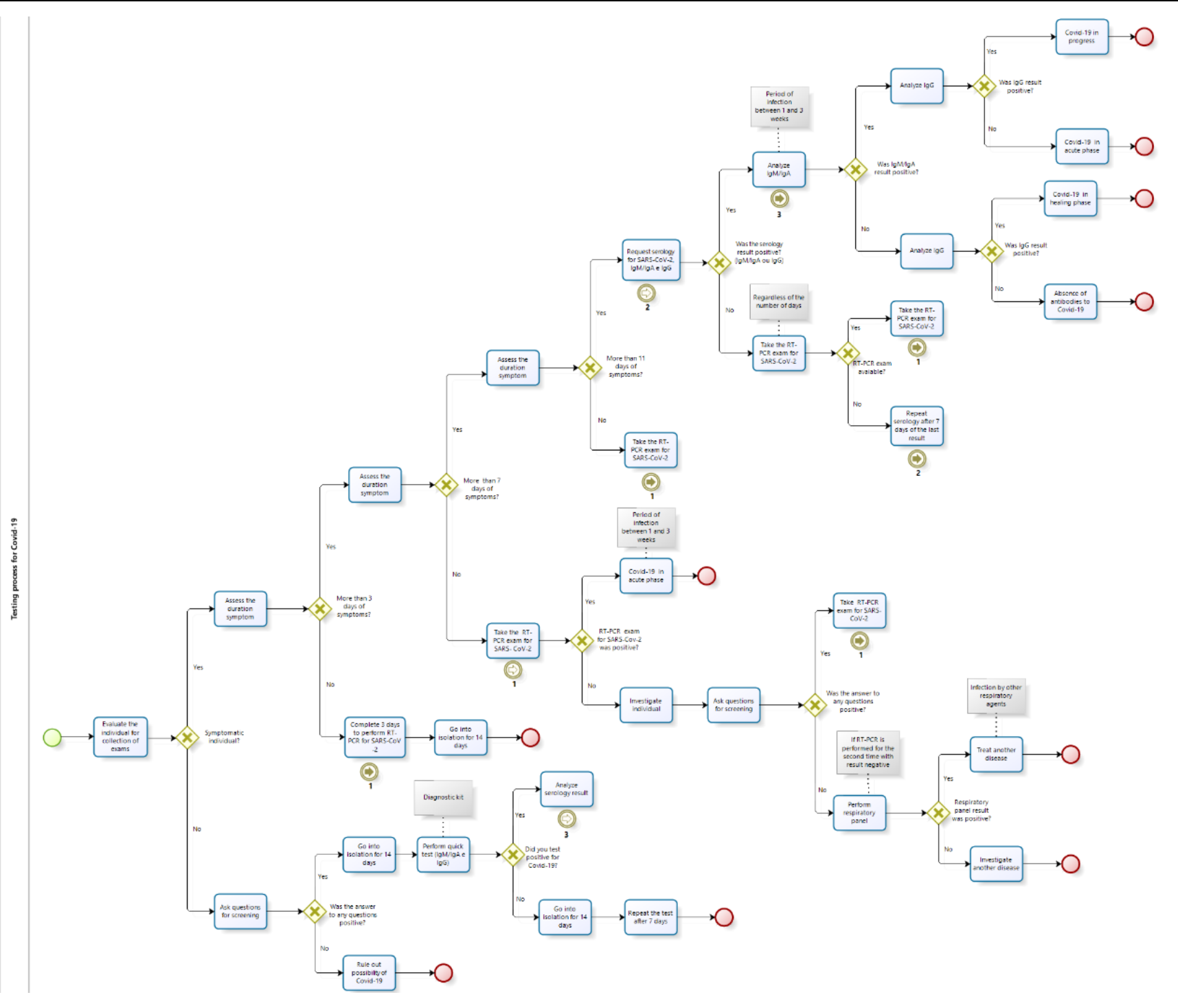

Figure 1. Test diagram. Source: Authors 
Following the flow of the symptomatic individual, it is necessary to assess the time that he has symptoms. If he has symptoms for more than three (3) days, ask if he has more than seven (7) days of symptoms. If so, ask if the individual has more than 11 days of symptoms; if positive, serology ought to be carried out to detect IgM or IgA and IgG antibodies.

Initially analyze the result of the serology, if it is positive, analyze the $\operatorname{IgM} / \operatorname{IgA}$, and if positive, the individual has COVID-19, and the infection period probably occurred between 1 and 3 weeks. If, in association with positive $\operatorname{IgM} / \operatorname{IgA}$, we have positive $\operatorname{IgG}$, it means that the individual has an ongoing infection, still has the potential for transmission, but is already at a later stage, as he already has seroconversion, from IgM / IgA to IgG. If found in association with positive $\operatorname{IgM} / \operatorname{IgA}$, negative IgG means that it is in the acute/initial phase of the disease. However, if the $\operatorname{IgM} / \operatorname{IgA}$ is negative, with only positive IgG, it is a sign that the individual has already had COVID-19 and is already in the healing phase, with the presence of only the memory antibody of the infection. It is important to note that the fact that a person has IgG positive does not mean that they have an immunity passport.

The discovery of this virus and the disease is relatively new, so the behavior of this immune response has not yet been fully elucidated. It is not known whether the response can be temporary or permanent and whether there is real immunity against a new infection.

If the result of the $\operatorname{IgM} / \operatorname{IgA}$ test is negative, and the $\operatorname{IgG}$ result is negative, it means that the individual does not have anti-SARS-CoV-2 antibodies. However, the individual cannot be considered to have had any contact with the virus, or it may be that the individual has the disease and has not yet manifested (seroconversion / presence of antibodies, as shown in Chart 1).

Table 1. Interpretation of IgM / IgA and IgG anti-SARS-CoV-2 Results for COVID-19

\begin{tabular}{|c|l|l|}
\hline \multicolumn{2}{|c|}{ Test result } & \multicolumn{1}{c|}{ Interpretation } \\
\hline IgM/IgA positive & IgG positive & $\begin{array}{l}\text { Has an ongoing infection, the individual has potential for } \\
\text { transmission, but is already in a late-stage, as he presents IgM / } \\
\text { IgA to IgG seroconversion }\end{array}$ \\
\hline IgM/IgA positive & IgG negative & \multicolumn{1}{c|}{ Has an acute, early-stage infection } \\
\hline IgM/IgA negative & IgG positive & $\begin{array}{l}\text { Individuals already had the disease. } \\
\text { Already have memory anti-SARS-CoV-2, but does not make it } \\
\text { an immunity passport }\end{array}$ \\
\hline IgM/IgA negative* & IgG negative* & $\begin{array}{l}\text { It does not have anti-SARS-CoV-2 } * \text { antibodies; However, the } \\
\text { individual cannot be taken into account to have had no contact } \\
\text { with the virus, or it may be that the individual has the disease and } \\
\text { has not yet manifested (seroconversion/presence of antibodies). }\end{array}$ \\
\hline
\end{tabular}

* A single serological result with negative antibodies does not rule out the possibility of current SARSCoV-2 infection or in the past

Source: Authors 
If the serology result is negative, the RT-PCR test must be performed (when available). The collection must be performed with a nasopharyngeal swab to complete this test and detect SARS-CoV-2.

If the RT-PCR is not available, and the individual has symptoms of COVID-19, it is recommended to collect blood to perform a new serology after 7 days, following the same criteria for analyzing the results, mentioned above.

If the individual has more than seven (7) days of symptoms and less than 11 days, or more than three (3) and less than seven (7) days of symptoms, RT-PCR must be carried out. If the result is positive, the individual has COVID-19, and the infection period probably occurred between 1 and 3 weeks. If the result is negative, it is interesting to investigate the individual, being necessary to answer some questions for screening, which will be like to point out below (the same ones used for asymptomatic patients).

If the answer to any of the screening questions is positive, it is recommended that you repeat the RT-PCR for SARS-CoV-2, as some factors may generate false-negative results, influenced by improper collection, packaging, or incorrect transportation of the material. If the result of the second test is also negative, and the individual still has symptoms, other etiologies should be investigated through the respiratory panel and/or diagnostic tests.

If the result of the respiratory panel is positive, the individual has an infection with other respiratory agents and must treat this disease, as he does not have COVID-19. If it is negative, the individual should investigate other diseases not contained in the respiratory panel, which have symptoms like COVID-19.

If the individual has had symptoms for less than three (3) days, it is advised that he wait at least until the third day to perform the collection for RT-PCR for SARS-CoV-2, but he must remain in isolation.

Technical guides report that the search for antibodies using serological tests can be performed after seven (7) days of symptoms; however, scientific research such as that by Liu et al (2020a; 2020b) and Zhao et al. (2020) showed that serological sensitivity varies from $50 \%$ to $90 \%$, when these tests are used after the 11th day of symptoms.

When monitoring the flow of the asymptomatic individual, some questions need to be asked for screening (regarding the actions that the individual performed in the last seven (7) days):

- Did you use public transportation, subway, or public bus?

- Did you are working outside the home?

- Did you have to go to school or university?

- Did you ever been to a crowded place?

- Did you leave for any trade?

- Did you visit an infected relative or friend?

- Did you ever have contact with an infected person?

- Do you have any idea where did you get the disease? 
If the answer to any of the screening questions is positive, the individual should be isolated for 14 days and perform a rapid antibody test (IgM / $\operatorname{IgA}$ or $\operatorname{IgG}$ ). If the rapid test was positive for COVID-19, the individual should analyze the result of the serology as mentioned above, to identify the stage of the disease.

If the test is negative, the individual must remain in isolation for 14 days and repeat the test after seven (7) days. If the answer to any of the screening questions is negative, discard the possibility of the individual being with COVID-19.

\section{Final considerations}

It can, therefore, be observed that with the contribution of the detection process (figure 1) and the results interpretation framework (table 1), this study contributes to a better clarification of the virus detection process via the available tests and the interpretation of the results. Results, assisting health professionals, clinics, laboratories, hospitals, and patients. The scientific community can also be contemplated from a procedural understanding, allowing advances in studies in other areas, such as computer science, mathematics, statistics, and engineering, which need binary input to advance in the construction of algorithms and systems. Thus, the objective of this research has been accomplished.

\section{Conflict of Interest}

The authors declare that the study was conducted in the absence of any commercial or financial relationships that could be interpreted as a potential conflict of interest.

\section{References}

Ho, C.S., Chee, C.Y., Ho, R.C. Mental Health Strategies to Combat the Psychological Impact of COVID19 Beyond Paranoia and Panic. Ann. Acad. Med. Singapore. 49, 1-3 (2020).

Kiernan, S., Bardi, J.S. (2020): UPDATED: Timeline of the Coronavirus, Available in (<https://www.thinkglobalhealth.org/article/updated-timeline-coronavirus >). [Access on June 25, 2020.]

Le, T. T., Andreadakis, Z., Kumar, A., Roman, R. G., Tollefsen, S., Saville, M., \& Mayhew, S. (2020). The COVID-19 vaccine development landscape. Nat Rev Drug Discov, 19(5), 305-306.

Liu, L., Liu, W., Zheng, Y., Jiang, X., Kou, G., Ding, J., ... \& Wu, W. (2020a). A preliminary study on serological assay for severe acute respiratory syndrome coronavirus 2 (SARS-CoV-2) in 238 admitted hospital patients. Microbes and Infection.

Liu, Y., Liu, Y., Diao, B., Ren, F., Wang, Y., Ding, J., \& Huang, Q. (2020b). Diagnostic Indexes of a Rapid IgG/IgM Combined Antibody Test for SARS-CoV-2. medRxiv. 2020; Available from https://doi.org/10.1101/2020.03.26.20044883

Mehta, P., McAuley, D. F., Brown, M., Sanchez, E., Tattersall, R. S., Manson, J. J., \& HLH Across Speciality Collaboration. (2020). COVID-19: consider cytokine storm syndromes and immunosuppression. Lancet (London, England), 395(10229), 1033.

Randhawa, G. S., Soltysiak, M. P., El Roz, H., de Souza, C. P., Hill, K. A., \& Kari, L. (2020). Machine learning using intrinsic genomic signatures for rapid classification of novel pathogens: COVID-19 case study. Plos one, 15(4), e0232391. 
Rothan, H. A., \& Byrareddy, S. N. (2020). The epidemiology and pathogenesis of coronavirus disease (COVID-19) outbreak. Journal of autoimmunity, 102433.

Velavan, T. P., \& Meyer, C. G. (2020). The COVID-19 epidemic. Tropical medicine \& international health, 25(3), 278.

Wang, D., Hu, B., Hu, C., Zhu, F., Liu, X., Zhang, J., ... \& Zhao, Y. (2020). Clinical characteristics of 138 hospitalized patients with 2019 novel coronavirus-infected pneumonia in Wuhan, China. JAMA [Internet]. 2020 Feb [cited 2020 Mar 23]; 323 (11): 1061-9.

World Health Organization (2020a). WHO Coronavirus Disease (COVID-19) Dashboard. Available in (<https://covid19.who.int/>). [Access in June 25, 2020.]

World Health Organization. (2020b). Coronavirus disease 2019 (COVID-19): situation report, 72.

Zhao J, Yuan Q, Wang H, Liu W, Liao X, Su Y, et al. Antibody responses to SARS-CoV-2 in patients of novel coronavirus disease 2019. medxriv [Internet]. 2020; Available from: https://www.medrxiv.org/content/10.1101/2020.03.02.20030189v1.full.pdf

Zheng, Y. Y., Ma, Y. T., Zhang, J. Y., \& Xie, X. (2020). COVID-19 and the cardiovascular system. Nature Reviews Cardiology, 17(5), 259-260.

Zhu, Z., Ph, D., Xu, S., Ph, D., Wang, H., Med, M., Liu, Z., Ph, D.: COVID-19 in Wuhan : Immediate Psychological Impact on 5062 Health Workers. medRxiv. (2020). 